ESAIM: PROCEEDINGS, October 2011, Vol. 32, p. 118-133

E. Cancès, N. Crouseilles, H. Guillard, B. Nkonga, and E. Sonnendrücker, Editors

\title{
AN AXISYMMETRIC PIC CODE BASED ON ISOGEOMETRIC ANALYSIS*
}

\author{
A. BacK $^{1}$, A. Crestetto ${ }^{1}$, A. Ratnani ${ }^{1}$ And E. SonnendrüCker ${ }^{1}$
}

\begin{abstract}
Isogeometric analysis has been developed recently to use basis functions resulting from the CAO description of the computational domain for the finite element spaces. The goal of this study is to develop an axisymmetric Finite Element PIC code in which specific spline Finite Elements are used to solve the Maxwell equations and the same spline functions serve as shape function for the particles. The computational domain itself is defined using splines or NURBS.
\end{abstract}

\section{INTRODUCTION}

The Vlasov equation describes the evolution of charged particles in an electromagnetic field, which can consist of an applied and a self-consistent field. The latter being computed using Maxwell's equations. So we consider the system of the Vlasov-Maxwell equations. Physical problems described by this system are varied and it is necessary to develop methods adapted for each one of them.

We are interested here in the emission of electrons in a diode with hemispherical cathode. The problem is a priori three-dimensional and we can use cylindrical coordinates $(z, r, \theta)$. Moreover this problem is such that the unknowns do not depend on $\theta$ so that we can assume $2 D$ axisymmetric geometry.

The IsoPIC code that we describe in this paper is a $(z, r)$ axisymmetric Vlasov-Maxwell solver.

Thanks to symmetry in $\theta$ direction, it is enough to give the description of the domain for a section (for example $\theta=0$ ). The isogeometric analysis approach will be applied, and we will construct a mapping $\mathbf{F}$ transforming a square into the section.

For the Maxwell equations, we consider in a first stage only the transverse electric mode (denoted by TE). The electric and magnetic field components considered are $E_{r}, E_{z}$ and $B_{\theta}$. To solve them, we use spline finite elements. This article begins with their definition, their properties and their application to solve our system.

Since their introduction, B-splines have had a large success because their implementation is based on fast and stable algorithms. They are used in industry and in academic research for interpolation, computer aided design and data fitting. Recent work by Hughes and his co-authors [14-16] and the introduction of isogeometric analysis added another dimension to their use, creating an interface between simulation and modeling.

Before the recent work of Hughes [14], the use of splines as basis functions in the finite element method was rare and mostly limited to uniform B-splines using periodic conditions, although the web-splines developed by Hoellig and others proposed a strategy for dealing with boundary conditions $[12,13]$. The idea of isogeometric analysis, using geometric transformations based splines and NURBS is easier for most applications. Compared with usual finite elements, the main change due to the isogeometric analysis is undoubtedly the emergence of the k-refinement, a strategy that can increase the regularity of functions with the interfaces of the mesh to reduce the number of degrees of freedom.

\footnotetext{
* The authors would like to acknowledge financial support by CEA Gramat and thank Mr Vézinet for helpful discussions

${ }^{1}$ IRMA, Université de Strasbourg and INRIA-Nancy-Grand Est, CALVI Project-Team
} 
Modern techniques of finite elements for Maxwell's equations are based on the ideas of differential geometry and more precisely the existence of discrete spaces that define an exact De Rham sequence. Following the pioneering ideas of Bossavit [5,6] a complete theory has been developed [2,11]. Buffa and al. [7, 8] have extended the isogeometric analysis to the steady state Maxwell's equations, providing a discrete exact De Rham sequence, in discrete spaces based on B-splines. The isogeometric analysis approach for studying unsteady Maxwell's equations has been studied by two of the authors [18].

The present article describes an axisymmetric Vlasov-Maxwell Particle In Cell (PIC) method based on isogeometric analysis. It is organized as follows: After recalling briefly the principles of isogeometric analysis, we introduce an axisymmetric Maxwell solver based on this approach. Then, we present the emission and the motion of particles in generalized coordinates on a patch. We study the conditions for extraction of particles in a diode with hemispherical cathode. And finally numerical results obtained with the code are proposed.

\section{IsOgeometric AnAlysis}

In this section, we give a brief description of using $B$-splines in a finite element method. More details can be found in $[14,16]$.

\subsection{B-Splines}

Let $T=\left(t_{i}\right)_{1 \leqslant i \leqslant N+k}$ be a non-decreasing sequence of knots.

Definition 2.1 (B-Spline). The $i$-th B-spline of order $k$ is defined by the recurrence relation:

$$
N_{j}^{k}=w_{j}^{k} N_{j}^{k-1}+\left(1-w_{j+1}^{k}\right) N_{j+1}^{k-1},
$$

where

$$
w_{j}^{k}(x)=\frac{x-t_{j}}{t_{j+k-1}-t_{j}}, \quad \quad N_{j}^{1}(x)=\chi_{\left[t_{j}, t_{j+1}[\right.}(x) .
$$

We note some important properties of a B-splines basis:

- B-splines are piecewise polynomial of degree $p=k-1$.

- Positivity.

- Compact support; the support of $N_{j}^{k}$ is contained in $\left[t_{j}, . ., t_{j+k}\right]$.

- Partition of unity: $\sum_{i=1}^{N} N_{i}^{k}(x)=1, \forall x \in \mathbb{R}$.

- Local linear independence.

- If a knot $t$ has a multiplicity $m$ then the B-spline is $\mathcal{C}^{(p-m)}$ at $t$.

Let $\left(P_{i}\right)_{1 \leqslant i \leqslant N} \in \mathbb{R}^{d}$ be a sequence of control points, forming a control polygon.

Definition 2.2 (B-Spline curve). The B-spline curve in $\mathbb{R}^{d}$ associated to $T=\left(t_{i}\right)_{1 \leqslant i \leqslant N+k}$ and $\left(P_{i}\right)_{1 \leqslant i \leqslant N}$ is defined by:

$$
\boldsymbol{M}(t)=\sum_{i=1}^{N} N_{i}^{k}(t) \boldsymbol{P}_{i} .
$$

Remark 2.3. In figure 1, we show an example of a B-spline curve. The basis here is simply Bernstein polynomials. In figure 2, we show the impact of inserting knots starting from a given knot vector. We remark the regularity of the new basis, for the repeated knot $\frac{3}{4}$. This refers as the k-refinement. The use of elements with high regularity at the interface of cells, allows us to reduce the dimension of the associated discrete space. More details on the impact of the k-refinement can be found in [10,14,18].

Remark 2.4. In order to use B-splines to treat $2 D$ or $3 D$ problems, we need to use multivariate B-splines, which can be constructed by a tensor product. This will generate a mapping $\mathbf{F}$ that maps a rectangular grid Patch, onto the physical domain $\Omega$. In figure 4, we show the impact of the h-refinement. Remark that using refinement strategies does not affect the mapping $\mathbf{F}$. 

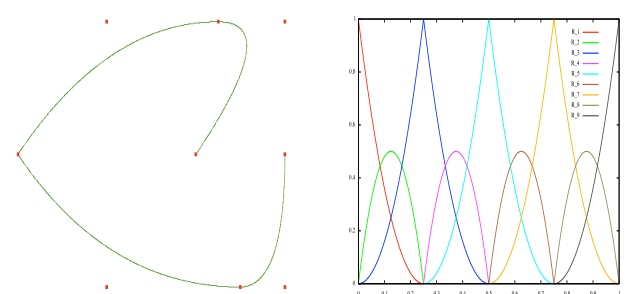

Figure 1. (left) A B-spline curve and its control points, (right) B-splines functions used to draw the curve. $N=9, p=2, T=\left\{000, \frac{1}{4} \frac{1}{4}, \frac{1}{2} \frac{1}{2}, \frac{3}{4} \frac{3}{4}, 111\right\}$.

\subsection{Grid generation}

Starting from a coarse grid, a new grid can be constructed by refinement, which can be done in three different ways:

- using the patch parameter $h$, by inserting new knots. This is the h-refinement, it is the equivalent of mesh refinement of the classical finite element method.

- using the degree $p$, by elevating the B-spline degree. This is the p-refinement, it is the equivalent of using higher finite element order in the classical FEM.

- using the regularity of B-splines, by increasing / decreasing the multiplicity of inserted knots. This is the $k$-refinement. This new strategy does not have an equivalent in the classical FEM.
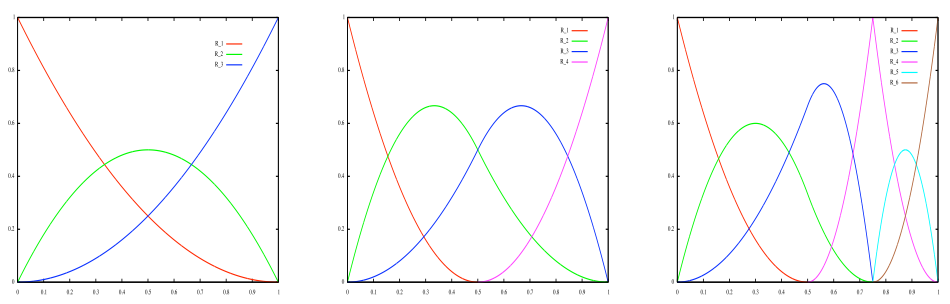

FigURE 2. Using h-refinement with $p=2, T=\{000,111\}, T=\left\{000, \frac{1}{2}, 111\right\}$ and $T=\left\{000, \frac{1}{2}, \frac{3}{4} \frac{3}{4}, 111\right\}$.

To define the computational mesh, we use alternatively $h$ and $p$-refinement, see figure 3 . The minimal degree of the basis functions is imposed by the domain design. When inserting knots, we can use uniformly-spaced knots or non uniformly-spaced ones.
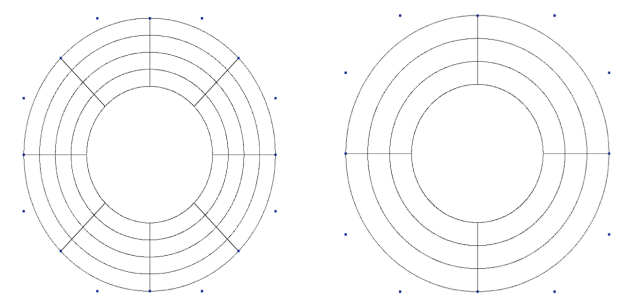

FiguRE 3. Grid generation. (left) after $h$-refinement, $N_{1}=17, N_{2}=5$, (right) after $p$ refinement, $p_{1}=p_{2}=3$. 


\subsection{Spline finite elements on general domains}

In order to use the spline finite elements on general domains, we shall use the ideas of isogeometric analysis and consider domains defined with NURBS that can be obtained via CAD software and use these NURBS to map a rectangular domain on the physical domain.

Let $Q$ be a cell in the physical domain. $\widetilde{Q}$ is the parametric associated cell and such that $Q=\mathbf{F}(\widetilde{Q})$. Let $J_{\mathbf{F}}$ be the Jacobian of the transformation $\mathbf{F}$, that maps any parametric domain point $(\xi, \eta)$ into physical domain point $(x, y)$.
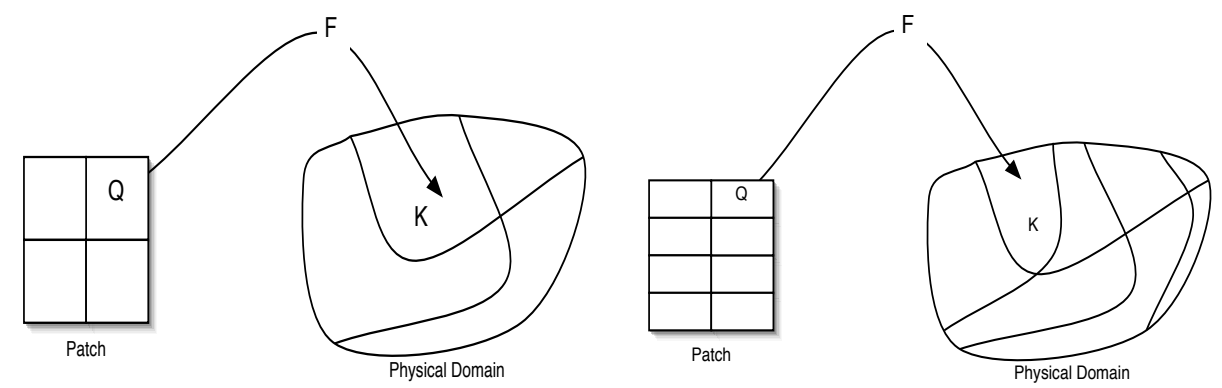

FiguRE 4. Mapping from the patch to the physical domain: (left) initial patch, (right) patch after h-refinement in the $\eta$ direction.

For any function $v$ of $(x, y)$, we associate its representation in the parametric domain

$$
\widetilde{v}((\xi, \eta))=v \circ \mathbf{F}((\xi, \eta))=v((x, y)) .
$$

The basis functions $R_{i}$ will not be affected by these changes, the reader can always know if we are working in the physical or parametric domain thanks to

$$
(x, y)=\mathbf{F}(\xi, \eta), x=\alpha(\xi, \eta) \text { and } y=\beta(\xi, \eta) .
$$

Then,

$$
\alpha_{1}=\frac{\partial \alpha}{\partial \xi}, \quad \alpha_{2}=\frac{\partial \alpha}{\partial \eta}, \quad \beta_{1}=\frac{\partial \beta}{\partial \xi}, \quad \beta_{2}=\frac{\partial \beta}{\partial \eta} .
$$

We have for the determinant of the Jacobian $\operatorname{det}\left(J_{\mathbf{F}}\right)=\alpha_{1} \beta_{2}-\alpha_{2} \beta_{1}$ and for $J_{\mathbf{F}^{-1}}$

$$
J_{\mathbf{F}}=\left(\begin{array}{cc}
\alpha_{1} & \alpha_{2} \\
\beta_{1} & \beta_{2}
\end{array}\right), \quad J_{\mathbf{F}^{-1}}=\frac{1}{\Delta}\left(\begin{array}{cc}
\beta_{2} & -\alpha_{2} \\
-\beta_{1} & \alpha_{1}
\end{array}\right) .
$$

Let $u$ be a (scalar or vector) function defined on the physical domain. When we use the patch coordinates, we will write it $\widetilde{u}$, idem for the used spaces.

\subsection{Boundary conditions}

The boundary conditions are very easy to handle. Thanks to the mapping, the boundary of the physical domain is mapped into the boundary of the patch. Periodic boundary conditions can be handled by using periodic splines with an adapted knot vector [4]. Perfect conductor and Silver-Müller boundary conditions are also implemented in a straighforward manner. 


\section{Axisymmetric Variational formulation of the 2D Maxwell equations}

The 2D Maxwell equations, in the TE mode, read

$$
\left\{\begin{array}{c}
-\frac{\partial \mathbf{E}}{\partial t}+\operatorname{curl} B=\mathbf{J} \\
\frac{\partial B}{\partial t}+\operatorname{curl} \mathbf{E}=0 \\
\operatorname{div} \mathbf{E}=\rho .
\end{array}\right.
$$

For the axisymmetric geometry, we have:

$$
\mathbf{E}=\left(\begin{array}{c}
E_{z} \\
E_{r}
\end{array}\right), B=B_{\theta}, \operatorname{curl} \mathbf{E}=\partial_{z} E_{r}-\partial_{r} E_{z}, \operatorname{div} \mathbf{E}=\frac{1}{r} \partial_{r}\left(r E_{r}\right)+\partial_{z} E_{z} \text { and } \operatorname{curl} B_{\theta}=\left(\begin{array}{c}
\frac{1}{r} \partial_{r}\left(r B_{\theta}\right) \\
-\partial_{z} B_{\theta}
\end{array}\right) .
$$

Variational formulation: As usual, to derive the variational formulation of the Maxwell equations, we will take the weak form of one equation (Ampère or Faraday), and keep the other one in the strong form. Multiplying each of the Maxwell equations by a test function and integrating on the physical domain $\Omega$, we obtain for the Ampère equation

$$
-\frac{\partial}{\partial t} \int_{\Omega} \mathbf{E} \cdot \boldsymbol{\Psi} d X+\int_{\Omega} \operatorname{curl} B \cdot \boldsymbol{\Psi} d X=\int_{\Omega} \mathbf{J} \cdot \boldsymbol{\Psi} d X, \quad \forall \boldsymbol{\Psi} \in H(\operatorname{div}, \Omega) .
$$

And, for the Faraday equation,

$$
\frac{\partial}{\partial t} \int_{\Omega} B \phi d X+\int_{\Omega} \operatorname{curl} \mathbf{E} \phi d X=0, \quad \forall \phi \in H^{1}(\Omega)
$$

Now by using the Green formula

$$
\int_{\Omega}(\operatorname{curl} G) \cdot \mathbf{F} d X=\int_{\Omega} G \operatorname{curl} \mathbf{F} d X-\int_{\Gamma}(G \times \mathbf{n}) \cdot \mathbf{F} d S, \forall \mathbf{F} \in H(\operatorname{curl}, \Omega), \forall G \in H^{1}(\Omega),
$$

and if we are dealing with perfectly conducting boundary conditions, we get

$$
\frac{\partial}{\partial t} \int_{\Omega} B \phi d X+\int_{\Omega} \mathbf{E} \cdot \operatorname{curl} \phi d X=0
$$

In the case of axisymmetric geometry, the measure $d X$ considered is simply $d X=r d r d z$.

Operators expressions: We have, $\mathbf{E} \cdot \boldsymbol{\Psi}=E_{z} \Psi_{z}+E_{r} \Psi_{r}, \mathbf{J} \cdot \mathbf{\Psi}=J_{z} \Psi_{z}+J_{r} \Psi_{r}, \mathbf{E} \cdot \operatorname{curl} \phi=E_{z} \frac{1}{r} \partial_{r}(r \phi)-$ $E_{r} \partial_{z} \phi$, and $\operatorname{curl} B \cdot \boldsymbol{\Psi}=\Psi_{z} \frac{1}{r} \partial_{r}(r B)-\Psi_{r} \partial_{z} B$. Therefore,

$$
-\frac{\partial}{\partial t} \int_{\Omega}\left(E_{z} \Psi_{z}+E_{r} \Psi_{r}\right) r d r d z+\int_{\Omega}\left(\Psi_{z} \partial_{r}(r B)-r \Psi_{r} \partial_{z} B\right) d r d z=\int_{\Omega}\left(J_{z} \Psi_{z}+J_{r} \Psi_{r}\right) r d r d z,
$$

and,

$$
\frac{\partial}{\partial t} \int_{\Omega} B \phi r d r d z+\int_{\Omega}\left(E_{z} \partial_{r}(r \phi)-r E_{r} \partial_{z} \phi\right) d r d z=0
$$

\subsection{Construction of the finite element spaces}

An important feature of the functional spaces we chose for the variational formulation is that they form an exact sequence. Depending on the variational formulation we choose, we need to work with different exact 
sequences. As we have relaxed the Faraday equation, the following function spaces are involved

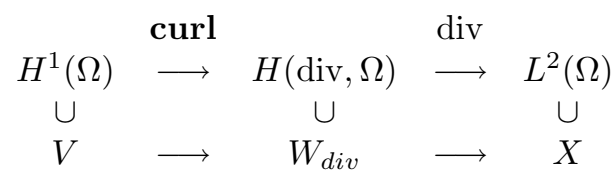

In order to keep the specific features of Maxwell's equations at the discrete level we need to construct finite dimensional subspaces endowed with the same structure. The involved discrete spaces are denoted by $V \subset H^{1}(\Omega), W_{d i v} \subset H(\operatorname{div}, \Omega)$ and $X \subset L^{2}(\Omega)$. In our case, we shall look for $\left(\mathbf{E}_{h}, H_{h}\right) \in W_{d i v} \times V$.

\subsection{Spline finite elements on patch grids}

We shall now start constructing the actual subspaces $V \subset H^{1}(\Omega), W_{\text {div }} \subset H(\operatorname{div}, \Omega)$ and $X \subset L^{2}(\Omega)$. Our discrete spaces will be constructed using B-splines. The key point of our method is the use of the recursion formula for the derivatives:

$$
N_{i}^{p^{\prime}}(t)=p\left(\frac{N_{i}^{p-1}(t)}{t_{i+p}-t_{i}}-\frac{N_{i+1}^{p-1}(t)}{t_{i+p+1}-t_{i+1}}\right) .
$$

It will be convenient to introduce the notation $D_{i}^{p}=p \frac{N_{i}^{p-1}(t)}{t_{i+p}-t_{i}}$. Then the recursion formula for derivatives (3) simply becomes

$$
N_{i}^{p^{\prime}}(t)=D_{i}^{p}(t)-D_{i+1}^{p}(t)
$$

Let us first consider a rectangular domain $\Omega$. We consider the following discrete functional spaces:

$$
\begin{gathered}
V=\operatorname{span}\left\{N_{i}^{p}(x) N_{j}^{p}(y), 1 \leq i \leq N_{x}, 1 \leq j \leq N_{y}\right\}, \\
\left.W_{d i v}=\operatorname{span}\left\{\left(\begin{array}{c}
N_{i}^{p}(x) D_{j}^{p}(y) \\
0
\end{array}\right),\left(\begin{array}{c}
0 \\
D_{i}^{p}(x) N_{j}^{p}(y)
\end{array}\right), \quad 1 \leq i \leq N_{x}, 1 \leq j \leq N_{y}\right\}\right\}, \\
X=\operatorname{span}\left\{D_{i}^{p}(x) D_{j}^{p}(y), 1 \leq i \leq N_{x}, 1 \leq j \leq N_{y}\right\} .
\end{gathered}
$$

As proved in the article by Buffa et al. [8], these spaces verify the same exact property as the spaces they approximate.

Let us now define coordinate changes conserving either the curl or the divergence of a vector field. Let us start from a vector field $\underline{\Psi}(\xi, \eta)=\left(\underline{\Psi}^{(1)}(\xi, \eta), \underline{\Psi}^{(2)}(\xi, \eta)\right)^{T}$ defined on the parametric domain $\tilde{Q}$. Using the transformation formula:

$$
\Psi=\left(\left(-\underline{\Psi}^{(2)} \circ G \frac{\partial G_{1}}{\partial y}+\underline{\Psi}^{(1)} \circ G \frac{\partial G_{2}}{\partial y}\right),\left(\underline{\Psi}^{(2)} \circ G \frac{\partial G_{1}}{\partial x}-\underline{\Psi}^{(1)} \circ G \frac{\partial G_{2}}{\partial x}\right)\right)^{T}
$$

for the vector fields of $W_{\text {div }}$ which conserves the divergence, using the diffeomorphism $G=F^{-1}$, we get $\Psi^{(1)}=\frac{1}{\Delta}\left(\alpha_{2} \Psi^{(2)}+\alpha_{1} \Psi^{(1)}\right)$, and $\Psi^{(2)}=\frac{1}{\Delta}\left(\beta_{2} \Psi^{(2)}+\beta_{1} \Psi^{(1)}\right)$.

So let $\Psi=\left(\Psi^{(1)}, \Psi^{(2)}\right)^{T}$ be a function in $W_{d i v}$, and $\underline{\Psi}=\left(\underline{\Psi}^{(1)}, \underline{\Psi}^{(2)}\right)^{T}$ be a function in $\widetilde{W}_{d i v}$. To preserve the divergence, the corresponding space of $\widetilde{W}_{d i v}$ on the physical domain is

$$
W_{d i v}=\left\{\Psi=\left(\frac{1}{\Delta}\left(\alpha_{1} \Psi^{(1)}+\alpha_{2} \Psi^{(2)}\right), \frac{1}{\Delta}\left(\beta_{1} \Psi^{(1)}+\beta_{2} \Psi^{(2)}\right)\right)^{T}, \quad \underline{\Psi} \in \widetilde{W}_{d i v}\right\}
$$

which can also be written

$$
W_{d i v}=\operatorname{span}\left\{\frac{1}{\Delta} \widetilde{N}_{i}^{p}(\xi) \widetilde{D}_{j}^{p}(\eta)\left(\begin{array}{c}
\alpha_{1} \\
\beta_{1}
\end{array}\right), \frac{1}{\Delta} \widetilde{D}_{i}^{p}(\xi) \widetilde{N}_{j}^{p}(\eta)\left(\begin{array}{c}
\alpha_{2} \\
\beta_{2}
\end{array}\right)\right\} .
$$




\subsection{Discrete equations}

Let $W_{d i v}=\operatorname{span}\left\{\vec{\psi}_{b}, b \in \Gamma_{E}\right\}$ the basis for $W_{d i v}$, the discrete space associated to the electric field. Let $V=\operatorname{span}\left\{\varphi_{b^{\prime}}, b^{\prime} \in \Gamma_{H}\right\}$ the basis of $V$, it discretizes the space associated to the magnetic field.

The mass matrices for the electric and magnetic field are respectively

$$
M_{b, b^{\prime}}^{W}=\int_{\Omega} \vec{\psi}_{b} \cdot \vec{\psi}_{b^{\prime}} r d r d z, \quad \text { and } M_{b, b^{\prime}}^{V}=\int_{\Omega} \varphi_{b} \varphi_{b^{\prime}} r d r d z
$$

The matrix for the curl is

$$
\begin{aligned}
K_{b, b^{\prime}}=\int_{\Omega} \operatorname{curl} \varphi_{b} \cdot & \vec{\psi}_{b^{\prime}} r d r d z=\int_{\Omega}\left(r \partial_{r} \varphi_{b}+\varphi_{b}\right) \psi_{b^{\prime}}^{z}-\left(r \partial_{z} \varphi_{b}\right) \psi_{b^{\prime}}^{r} d r d z \\
& =\int_{\Omega} r\left(\partial_{r} \varphi_{b} \psi_{b^{\prime}}^{z}-\partial_{z} \varphi_{b} \psi_{b^{\prime}}^{r}\right) d r d z+\int_{\Omega} \varphi_{b} \psi_{b^{\prime}}^{z} d r d z
\end{aligned}
$$

This is the contribution of two terms. The first one " $r\left(\partial_{r} \varphi_{b} \psi_{b^{\prime}}^{z}-\partial_{z} \varphi_{b} \psi_{b^{\prime}}^{r}\right)$ " which differs from the cartesian formulation by a multiplication by $r$. The second one " $\varphi_{b} \psi_{b^{\prime}}^{z}$ " is due to the axisymmetric geometry.

\subsection{Domain parametrization using Splines/NURBS curves}

The parametrization of the domain by curves defined with aid of B-splines and NURBS is a recent topic. Indeed, it is about creating the mesh of the boundary of the domain from a description in terms of curves. Few articles that discuss the topic provide strategies to build these meshes $[1,9,17,20]$.

In our case, the problem is different. We must be able to create a mesh from the description of the boundary and this mapping must be $\mathcal{C}^{1}$.

The easiest approach, which involves using NURBS to describe the quarter of circle of the diode, leads to a mapping which is continuous at the crossing of some nodes and not $\mathcal{C}^{1}$, see Fig. 5. We thus opted for a version in the spirit isoparametric analysis using only B-splines. The elements used to describe the boundary improve this description by increasing the degree of splines. A quarter of the circle is constructed by interpolation, by choosing a degree, determined by the user.

Remark 3.1. In our numerical tests, we have token the weights equal to 1, and changed the knot vector $T=\left\{\begin{array}{llllll}000 & \frac{1}{3} & \frac{1}{3} & \frac{2}{3} & 2 & 111\end{array}\right\}$ into $T=\left\{\begin{array}{llllll}000 & 0.2 & 0.4 & 0.6 & 0.8 & 111\end{array}\right\}$, in order to have a mapping which is $\mathcal{C}^{1}$.
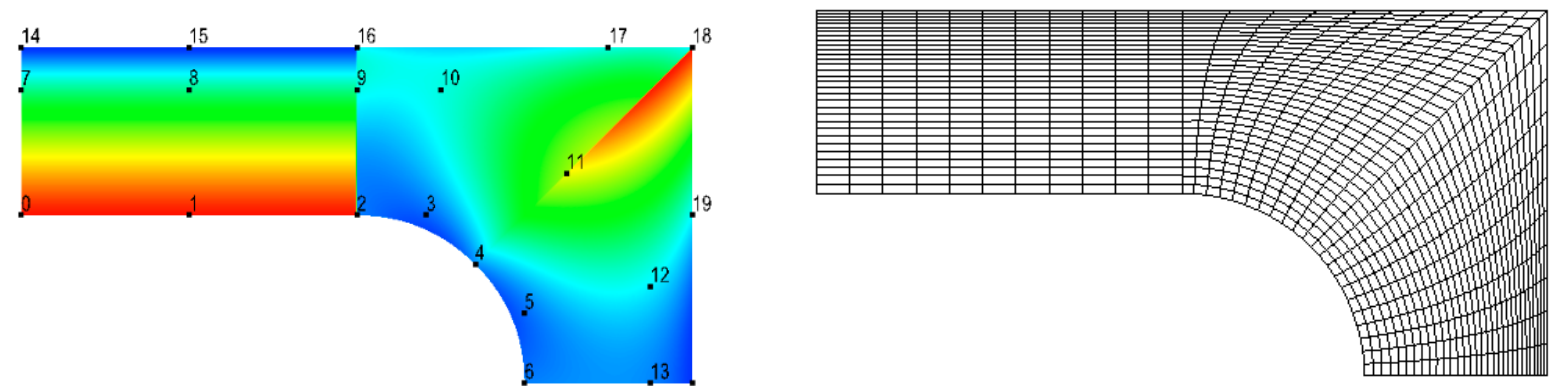

Figure 5. Diode: (left) the Jacobian of the mapping and the control points, (right) the mesh.

Remark 3.2. In figure 8, we give the description used to construct the domain, using NURBS. 
A version of the domain, obtained by keeping the control points and weights equal to 1 (in which case the NURBS become B-splines), is presented later. This domain is used for validation, before looking a more advanced version.

\section{PIC Method FOR Vlasov EQUATion}

All the equations are solved on the patch. Since we have to change coordinates to map the physical domain into the patch, we must compute the Maxwell equations, the equations of motion, the densities of current and charge and the emission of particles in a general coordinates system.

\subsection{The PIC Method}

To solve the Vlasov equation

$$
\frac{\partial f}{\partial t}+\mathbf{V} \cdot \nabla_{X} f-(\mathbf{E}+\mathbf{V} \wedge B) \cdot \nabla_{V} f=0
$$

we use the PIC method [19], [3]. We consider a set of $N$ macro-particles with $\mathbf{X}_{\mathbf{k}}$ being as the position, $\mathbf{V}_{\mathbf{k}}$ as the velocity, and $\omega_{k}$ as the weight. The particles represent the distribution function $f$. We approach $f$ by a sum of Dirac functions centered in the positions and in the velocities of particles:

$$
f(\mathbf{X}, \mathbf{V}, t) \approx f_{N}(\mathbf{X}, \mathbf{V}, t)=\sum_{k=1}^{N} \omega_{k} \delta\left(\mathbf{X}-\mathbf{X}_{\mathbf{k}}(t)\right) \delta\left(\mathbf{V}-\mathbf{V}_{\mathbf{k}}(t)\right) .
$$

The motion of particles is described by the equations of motion in which the electric and magnetic fields are used. They are obtained by solving the Maxwell equations which involves the charge density $\rho$ and the current density $\mathbf{J}$. We present the different steps of the PIC method:

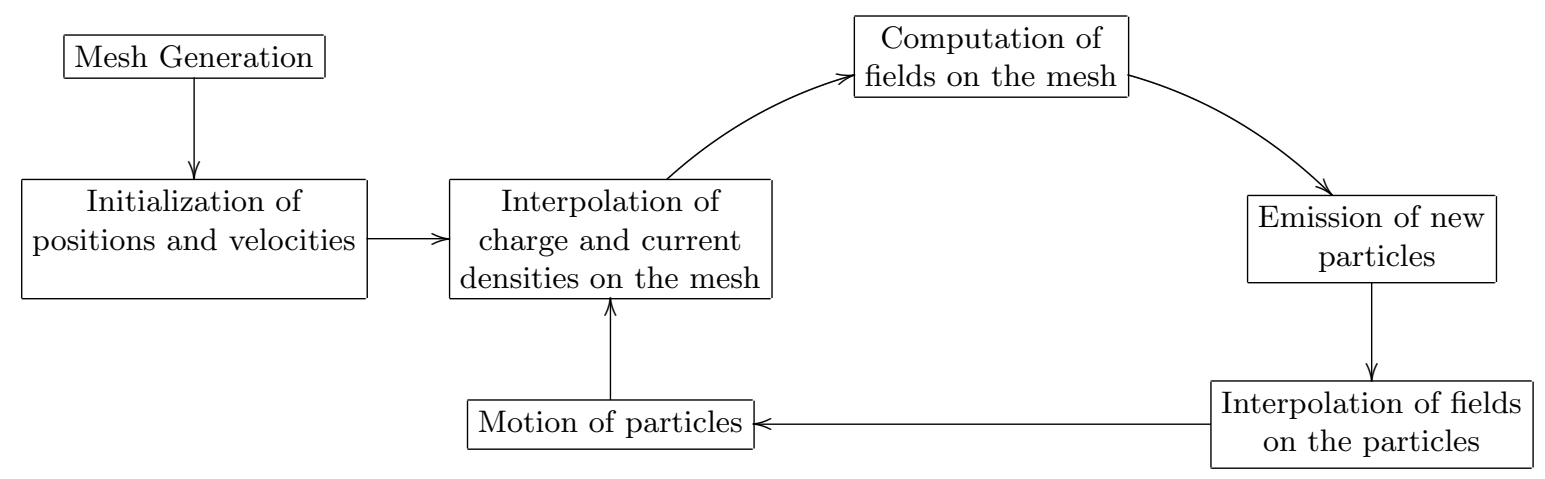

The most important steps that we develop in the following are:

- the description of equations of motion,

- the computation of charge density and current density,

- the condition for the emission of the particles.

\subsection{The equations of motion}

In our case, we move the particles in the patch, because it is easier to localize the particles on it. Let us explain how to obtain the equations of motion in generalized coordinates. 
The equations of motion in Lagrangian mechanics are the Lagrange equations, also known as the EulerLagrange equations. There are available in any coordinate system:

$$
\frac{d}{d t} \frac{\partial L}{\partial \dot{\mathbf{q}}}(\mathbf{q}, \dot{\mathbf{q}}, t)=\frac{\partial L}{\partial \mathbf{q}}(\mathbf{q}, \dot{\mathbf{q}}, t)
$$

where $L(\mathbf{q}, \dot{\mathbf{q}}, t)$ is the Lagrangian.

To write and solve the equations of motion in any coordinate system, we must know the Lagrangian to obtain the Lagrange equations. We start from its expression in cylindrical coordinates, where denoting by $\mathbf{A}$ the vector potential and $\phi$ the scalar potential, it has the form

$$
L(z, r, \theta, \dot{z}, \dot{r}, \dot{\theta}, t)=\frac{1}{2} m\left(\dot{r}^{2}+r^{2} \dot{\theta}^{2}+\dot{z}^{2}\right)-e\left(A_{r} \dot{r}+A_{\theta} \dot{\theta}+A_{z} \dot{z}-\phi(z, r, \theta)\right) .
$$

Now, we define the map $F$ which transforms the cylindrical coordinates $F(\xi, \eta, \theta)=(z, r, \theta)$ into a new coordinate system $(\xi, \eta, \theta)$. It is denoted by $F(\xi, \eta, \theta)=(z, r, \theta)$. But, since we work in $2 D$ axisymmetric geometry, we have $\dot{\theta}=0$, and so the map is simplified by $F(\xi, \eta)=(z, r)$.

In this coordinate system, the Lagrangian has the form

$$
L(\xi, \eta, \theta, \dot{\xi}, \dot{\eta}, \dot{\theta}, t)=\frac{1}{2} m\left(M_{\xi} \dot{\xi}^{2}+M_{\eta} \dot{\eta}^{2}+2 M_{\xi \eta} \dot{\xi} \dot{\eta}\right)-e\left(A_{\xi} \dot{\xi}+A_{\eta} \dot{\eta}-\phi(\xi, \eta, \theta)\right),
$$

with $\left(A_{\xi}, A_{\eta}, A_{\theta}\right)$ the components of the vector potential in the coordinate system $(\xi, \eta, \theta)$ and with

$$
M_{\xi}=\left(\frac{\partial r}{\partial \xi}\right)^{2}+\left(\frac{\partial z}{\partial \xi}\right)^{2}, \quad M_{\eta}=\left(\frac{\partial r}{\partial \eta}\right)^{2}+\left(\frac{\partial z}{\partial \eta}\right)^{2}, \quad M_{\xi \eta}=\frac{\partial r}{\partial \xi} \frac{\partial r}{\partial \eta}+\frac{\partial z}{\partial \xi} \frac{\partial z}{\partial \eta} .
$$

From this new Lagrangian, we can deduce the equations of motion in the new coordinate system:

$$
\begin{aligned}
\operatorname{det}(J) \frac{d \dot{\xi}}{d t}+\dot{\xi}^{2} K_{\xi, \eta}+\dot{\eta}^{2} K_{\eta, \eta}+2 \dot{\eta} \dot{\xi} K_{\eta \xi, \eta} & \left.=-\frac{e}{m \operatorname{det}(J)}\left(\left(\left.(\mathbf{E}+\dot{\mathbf{q}} \wedge \mathbf{B})\right|_{\xi}\right) M_{\eta}-\left.(\mathbf{E}+\dot{\mathbf{q}} \wedge \mathbf{B})\right|_{\eta}\right) M_{\xi \eta}\right) \\
\operatorname{det}(J) \frac{d \dot{\eta}}{d t}-\dot{\xi}^{2} K_{\xi, \xi}-\dot{\eta}^{2} K_{\eta, \xi}-2 \dot{\eta} \dot{\xi} K_{\xi \eta, \xi} & \left.=-\frac{e}{m \operatorname{det}(J)}\left(\left(\left.(\mathbf{E}+\dot{\mathbf{q}} \wedge \mathbf{B})\right|_{\eta}\right) M_{\xi}-\left.(\mathbf{E}+\dot{\mathbf{q}} \wedge \mathbf{B})\right|_{\xi}\right) M_{\xi \eta}\right)
\end{aligned}
$$

with

$$
K_{\xi, \xi}=H_{\xi} V_{\xi}, \quad K_{\xi, \eta}=H_{\xi} V_{\eta}, \quad K_{\eta, \eta}=H_{\eta} V_{\eta}, \quad K_{\eta, \xi}=H_{\eta} V_{\xi}, \quad K_{\xi \eta, \xi}=H_{\xi \eta} V_{\xi}, \quad K_{\xi \eta, \eta}=H_{\xi \eta} V_{\eta},
$$

where

$$
H_{\xi}=\left(\begin{array}{c}
\frac{\partial^{2} z}{\partial^{2} \xi} \\
\frac{\partial^{2} r}{\partial^{2} \xi}
\end{array}\right), \quad H_{\eta}=\left(\begin{array}{c}
\frac{\partial^{2} z}{\partial^{2} \eta} \\
\frac{\partial^{2} r}{\partial^{2} \eta}
\end{array}\right), \quad H_{\xi \eta}=\left(\begin{array}{c}
\frac{\partial^{2} z}{\partial \xi \partial \eta} \\
\frac{\partial^{2} r}{\partial \xi \partial \eta}
\end{array}\right), \quad V_{\xi}=\left(\begin{array}{c}
\frac{\partial r}{\partial \xi} \\
-\frac{\partial z}{\partial \xi}
\end{array}\right), \quad V_{\eta}=\left(\begin{array}{c}
\frac{\partial r}{\partial \eta} \\
-\frac{\partial z}{\partial \eta}
\end{array}\right) .
$$

$\operatorname{det}(J)=\frac{\partial z}{\partial \xi} \frac{\partial r}{\partial \eta}-\frac{\partial z}{\partial \eta} \frac{\partial r}{\partial \xi}$ is the Jacobian of the change of coordinates $F$, the components of $\mathbf{E}, \mathbf{B}$ are the components of the electric field and the magnetic field in $(\xi, \eta, \theta)$ and $\dot{\mathbf{q}}=\left(\begin{array}{c}\dot{\xi} \\ \dot{\eta}\end{array}\right)$.

Numerically, we consider $\dot{\xi}$ and $\dot{\eta}$ as independent variables with $\dot{\xi}=\frac{d \xi}{d t}$ and $\dot{\eta}=\frac{d \eta}{d t}$. We then solve the resulting first order system of ordinary differential equations using the second-order Runge-Kutta method. 


\subsection{The Dirac mass with a change of variables}

The Dirac mass at a point $\mathbf{x}_{k}$ is denoted by $\delta\left(\mathbf{x}-\mathbf{x}_{k}\right)$. It has the following properties:

$$
1=\int_{M} \delta\left(\mathbf{x}-\mathbf{x}_{\mathbf{k}}\right) d \mathbf{x}, \quad \text { and } g\left(\mathbf{x}_{\mathbf{k}}\right)=\int_{M} \delta\left(\mathbf{x}-\mathbf{x}_{\mathbf{k}}\right) g(\mathbf{x}) d \mathbf{x}, \quad \text { for any continuous function } g .
$$

Let us consider the change of coordinates $F(\xi, \eta)=\mathbf{x}, M$ is a grid of the physical domain such as $F(\tilde{M})=M$, and $\operatorname{Jac}(\xi, \eta)$ the jacobian of $F$. Since the integral value does not change when we change the coordinates we can deduce that

$$
1=\int_{M} \delta\left(\mathbf{x}-\mathbf{x}_{k}\right) d \mathbf{x}=\int_{\tilde{M}} \delta\left(F(\xi, \eta)-\mathbf{x}_{k}\right) \operatorname{Jac}(\xi, \eta) d \xi d \eta=\int_{\tilde{M}} \frac{\delta\left(\xi-\xi_{k}\right) \delta\left(\eta-\eta_{k}\right)}{J a c\left(\xi_{k}, \eta_{k}\right)} J a c(\xi, \eta) d \xi d \eta
$$

with $F\left(\xi_{k}, \eta_{k}\right)=\mathbf{x}_{\mathbf{k}}$ and because by definition $\delta\left(F(\xi, \eta)-\mathbf{x}_{k}\right)=\frac{\delta\left(\xi-\xi_{\mathbf{k}}\right) \delta\left(\eta-\eta_{k}\right)}{J a c\left(\xi_{\mathbf{k}}, \eta_{k}\right)}$, so

$$
g\left(\mathbf{x}_{\mathbf{k}}\right)=\int_{M} \delta\left(\mathbf{x}-\mathbf{x}_{k}\right) g(\mathbf{x}) d \mathbf{x}=\int_{\tilde{M}} g(F(\xi, \eta)) \delta\left(F(\xi, \eta)-\mathbf{x}_{k}\right) J a c(\xi, \eta) d \xi d \eta=g\left(F\left(\xi_{k}, \eta_{k}\right)\right) .
$$

\subsection{Computing $J$ and $\rho$ with a change of variables}

In the physical domain, we have $\rho(\mathbf{X}, t)=-\int_{\mathbf{V}} f(\mathbf{X}, \mathbf{V}, t) d \mathbf{V}$, and $\mathbf{J}(\mathbf{X}, t)=-\int_{\mathbf{V}} f(\mathbf{X}, \mathbf{V}, t) \mathbf{V} d \mathbf{V}$. Replacing $f$ by its sum of Dirac function (5), we have:

$$
\begin{aligned}
& \rho(\mathbf{X}, t)=-\sum_{k=1}^{N} \omega_{k} \delta\left(\mathbf{X}-\mathbf{X}_{\mathbf{k}}(t)\right) \\
& \mathbf{J}(\mathbf{X}, t)=-\sum_{k=1}^{N} \omega_{k} \mathbf{V}_{\mathbf{k}}(t) \delta\left(\mathbf{X}-\mathbf{X}_{\mathbf{k}}(t)\right) .
\end{aligned}
$$

Numerically, we have to compute the integral of these functions in space. It is easier to do it on the patch. With help of previous part and the equality (6), we deduce

$$
\int_{M} \mathbf{J}(\mathbf{X}, t) \cdot \psi d \mathbf{X}=\int_{\tilde{M}} \sum_{k} \omega_{k} \delta\left(F(\xi, \eta)-\mathbf{X}_{\mathbf{k}}(t)\right) \mathbf{V}_{\mathbf{k}}(t) J a c(\xi, \eta) d \xi d \eta=\sum_{k \mid \mathbf{X}_{\mathbf{k}}(t) \in M} \omega_{k} \mathbf{V}_{\mathbf{k}}(t) \cdot \psi\left(\mathbf{X}_{\mathbf{k}}(t)\right)
$$

and

$$
\int_{M} \rho(\mathbf{X}, t) \varphi d \mathbf{X}=\int_{\tilde{M}} \sum_{k} \omega_{k} \delta\left(F(\xi, \eta)-\mathbf{X}_{\mathbf{k}}(t)\right) \operatorname{Jac}(\xi, \eta) d \xi d \eta=\sum_{k \mid \mathbf{X}_{\mathbf{k}}(t) \in M} \omega_{k} \varphi\left(\mathbf{X}_{\mathbf{k}}(t)\right),
$$

where $M$ is a cell of a physical domain and $\tilde{M}$ a cell in the patch such as $F(\tilde{M})=M$.

\section{Particles EMission}

\subsection{Short description of a diode}

A diode is constituted of two semiconductors: a cathode rich in electrons and an anode which lacks them. If we apply a positive tension at the anode and a negative one at the cathode, such that the created potential drop is greater than a threshold value, electrons are extracted of the cathode and move towards the anode. This phenomena allows an electric current to pass. The movement of electrons near the cathode can create a vicious circle: they increase the potential drop, allowing it to propagate in the diode, and new electrons are extracted and moved. 
Numerically, we can impose a negative electric field at the entry of the diode and allow it to propagate. If it is strong enough, it extracts electrons of the cathode, they go towards the anode. Our domain is meshed, so we look at each cell to see if the field satisfies the conditions of the emission of particles.

\subsection{Extraction conditions}

At a given frequency, particles are created in each cell $\Omega$ respecting the following conditions:

- the cell $\Omega$ touches the cathode,

- the normal electric field to the cathode on $\Omega$ is greater than a threshold value.

Weights of created particles are positive and such that the following relation is respected: $\operatorname{div} \mathbf{E}=\rho$, and in the same time, the normal electric field to the cathode is zero according to the Child-Langmuir law. We integrate this relation on a cell $\Omega$ :

$$
\int_{\Omega} \operatorname{div} \mathbf{E} d \Gamma=\int_{\Omega} \rho d \Gamma
$$

The Stokes formula leads to: $\int_{\Omega} \operatorname{div} \mathbf{E} d \Gamma=\int_{\partial \Omega} \mathbf{E} \cdot \mathbf{n} d \gamma$. Besides, the approximation (5) gives:

$$
\int_{\Omega} \rho d \Gamma=\sum_{\text {particle }} \in \Omega=\omega_{k}
$$

because particles are electrons, their charge is negative but their weight is positive. We obtain the relation

$$
\int_{\partial \Omega} \mathbf{E} \cdot \mathbf{n} d \gamma=\int_{\partial \Omega_{0}} \mathbf{E} \cdot \mathbf{n} d \gamma+\int_{\partial \Omega_{1}} \mathbf{E} \cdot \mathbf{n} d \gamma=\sum_{\text {particle } k \in \Omega}-\omega_{k},
$$

where $\partial \Omega_{0}$ is the boundary of $\Omega$ touching the cathode and $\partial \Omega_{1}$ is the union of other boundaries of $\Omega$. We want to make $\int_{\partial \Omega_{0}} \mathbf{E} \cdot \mathbf{n} d \gamma$ vanish, the weight of injected particles is imposed such that we have

$$
\int_{\partial \Omega_{1}} \mathbf{E} \cdot \mathbf{n} d \gamma=\sum_{\text {particle }}-\omega_{k}
$$

\section{Numerical RESults}

\subsection{Validation of the Maxwell solver}

\subsubsection{Verification of the order of convergence}

We test the order of convergence of the Maxwell solver on a square in axisymmetric geometry. Using the classical FEM discretization, the expected theoretical order for a refinement of order $p$ is $p+1$ for the magnetic field and $p$ for the electric field.

Remark 6.1. We have noticed that we do not get this order using $\boldsymbol{H}$-div formulation. The order obtained is more of the form:

- the convergence order for $B$ is :

$$
\begin{cases}p-\frac{1}{2}, & \text { if } p \text { is even } \\ p+\frac{1}{2}, & \text { if } p \text { is odd }\end{cases}
$$


- the convergence order for $E_{z}$ is $p+1$,

- the convergence order for $E_{r}$ is the minimum of the two previous ones.

However, using $\boldsymbol{H}$-rot formulation we recover the classical order.

We solve numerically the Maxwell equations on a rectangle $z \in(0, L)$ and $r \in(0, R)$. Let $m$ be the mode, the analytic solution is

$$
\begin{gathered}
H(z, r)=\frac{\omega}{c} \frac{\lambda_{p}}{R} \sqrt{\frac{\epsilon_{0}}{\mu_{0}}} J_{1}\left(\frac{\lambda_{p}}{R} r\right) \cos \left(\frac{m \pi z}{L}\right) \cos (\omega t), \\
E^{z}(z, r)=\frac{1}{\mu_{0}}\left(\frac{\lambda_{p}}{R}\right)^{2} J_{0}\left(\frac{\lambda_{p}}{R} r\right) \cos \left(\frac{m \pi z}{L}\right) \sin (\omega t), \\
E^{r}(z, r)=\frac{1}{\mu_{0}} \frac{m \pi}{L} \frac{\lambda_{p}}{R} J_{1}\left(\frac{\lambda_{p}}{R} r\right) \sin \left(\frac{m \pi z}{L}\right) \sin (\omega t),
\end{gathered}
$$

where $J_{n}$ are Bessel functions of the first kind order $n$. $\lambda_{p}$ is the $p^{t h}$ zero of $J_{0}$.

We plot the error in L2 norm between the computed solution and the analytic solution based on the maximum diameter of the mesh, denoted by $\mathrm{h}$. For the tests we take $[0,1] \times[0,1]$ for the domain and $N$ the number of cells equal to $4,8,16,32$ and 64 . The time step is $h \times 10^{-5}$, small enough so that the order of the time scheme, which is 2 in our case, does not matter. We perform respectively 25, 50,100, 200 and 400 iterations. The $p$-refinement is between degrees 0 and 3. We add the degree used for the domain, here it is equal to 1 . The curves are represented with logarithmic scale in the figure 6.

\subsubsection{Emission of particles in the diode}

We studied qualitatively the extraction of particles in the domain representing the diode. We impose at the beginning of the diode a tension that increases linearly until reaching a threshold value, and is then constant. Particles are emitted at the cathode under the conditions of extraction described previously. We take the time step $d t=1.0 \times 10^{-2}$ and perform 25000 iterations. We represent the electric field $E_{r}$ and the particles at the time $\mathrm{T}=25,50,75,100,125,150,175$ and 200 in the figure 7 . 

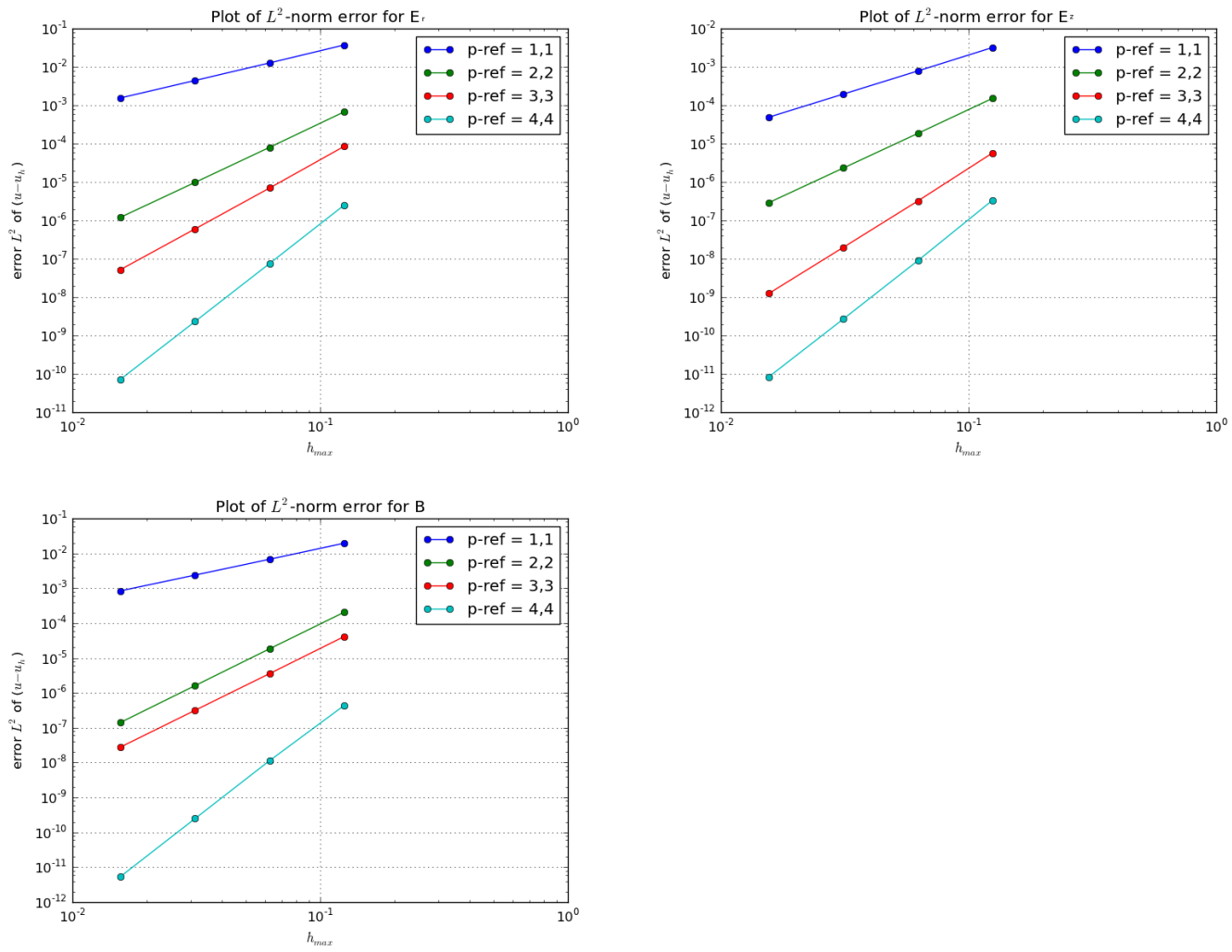

FiguRE 6. $L_{2}$ norm for the electric fields $E_{z}, E_{r}$ and the magnetic field $B_{\theta}$, for splines with degrees $1,2,3,4$.

\section{Conclusion And PeRspectives}

We have developed the IsoPIC code which solves the Vlasov-Maxwell equations in $2 D$ axisymmetric geometry. It is based on isogeometric analysis. It is applied to emit electrons in a diode with a hemispherical cathode, which we approximated using splines. The solution of the Vlasov equation is performed with a PIC method by moving the particles emitted at the cathode.

The Maxwell solver in axisymmetric geometry gives good results. The theoretical orders of convergence are checked on a square and the fields evolve correctly in the diode. Qualitatively, we also have good results for the particles. The extraction seems to be good but we have to add a confinement for which the TM mode, we have not implemented up to now, is needed.

We noticed that the conjugate gradient method is not good for our problem because the mass matrix of the electric field in axisymmetric geometry is not well conditioned and this algorithm does not converge fast enough. We did not notice this problem in cartesian coordinates where the matrices are better conditioned. So we used the direct solver Pastix, which has the advantage to be faster than iterative solvers for this kind of problems in $2 \mathrm{D}$.

The equations of motion are written and implemented in a general coordinate system. We have tested them in particular cases and we have compared the results with polar coordinates. The use of generalized coordinates is a choice for this study, despite the bigger calculation time. We would like to test later other possibilities, like cartesian coordinates with a mapping/inverse mapping to handle the mesh. 

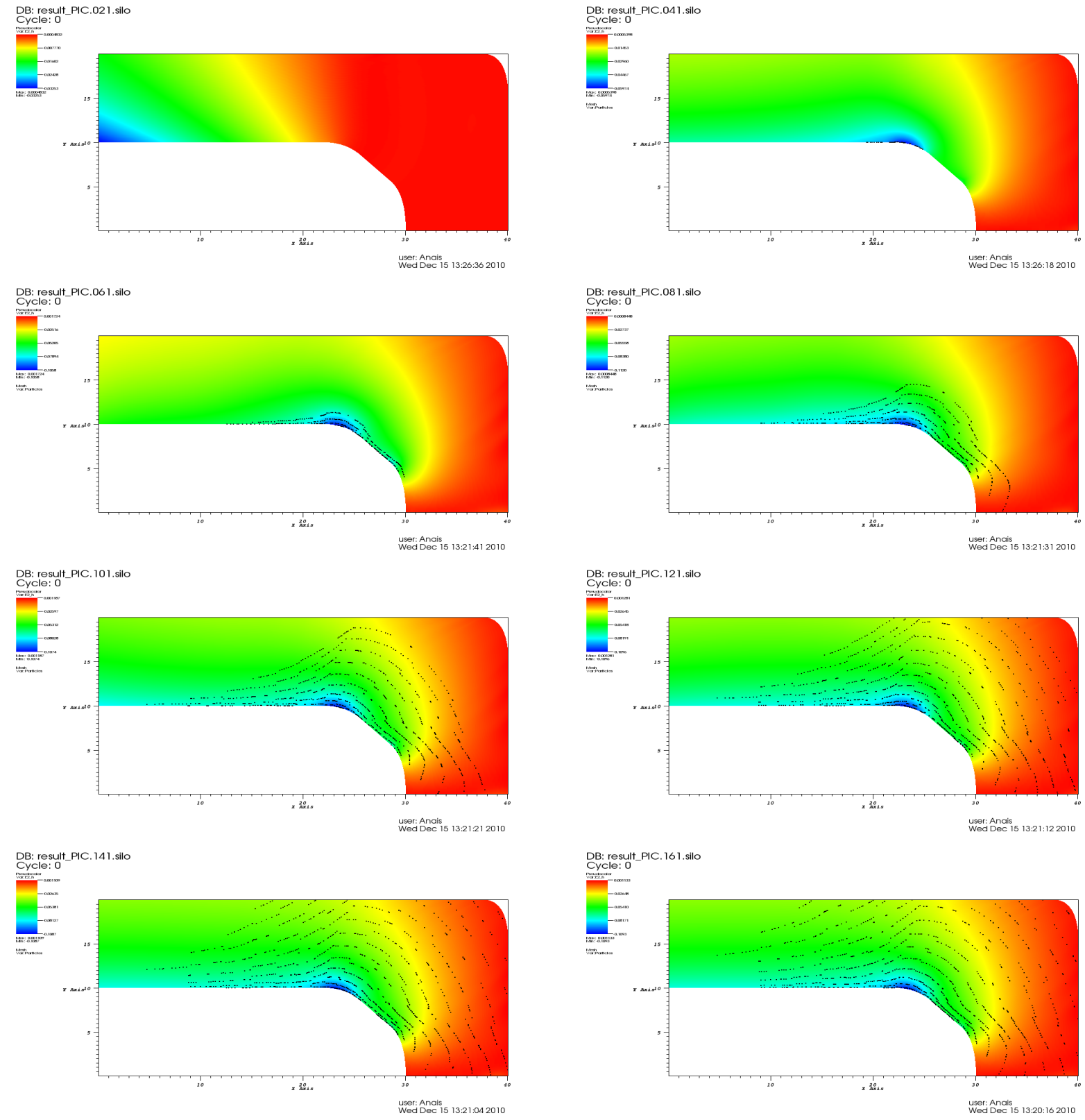

Figure 7. Electric field and electrons at time $\mathrm{T}=25, \mathrm{~T}=50$ (first line), $\mathrm{T}=75, \mathrm{~T}=100$ (second line), $\mathrm{T}=125, \mathrm{~T}=150$ (third line), $\mathrm{T}=175$ and $\mathrm{T}=200$ (last line).

This work needs to be continued addressing the following topics. On the one hand, we can rewrite the Maxwell equations in order to not have to invert the mass matrix of the electric field. On the other hand, we have to compute a Poisson solver, in order to consider more general cases. In fact, if there are particles at initial time (which is not the case in this study), we have to solve the Poisson equation initially. Then, the conservation of charge is obtained thanks to the exact De Rham sequence. Finally, we can parallelize the Maxwell solver 
and the motion of particles using GPU (Graphics Processing Unit), to reduce computation time. This kind of parallelization is indeed very efficient in our costly computations, which do not require a lot of memory.

\section{REFERENCES}

[1] M. Aigner, C. Heinrich, B. Jüttler, E. Pilgerstorfer, B. Simeon, and A. V. Vuong. Swept volume parameterization for isogeometric analysis. In Proceedings of the 13th IMA International Conference on Mathematics of Surfaces XIII, pages 19-44, Berlin, Heidelberg, 2009. Springer-Verlag.

[2] Douglas N. Arnold, Richard S. Falk, and Ragnar Winther. Finite element exterior calculus, homological techniques, and applications. Acta Numer., 15:1-155, 2006.

[3] C. K. Birdsall and A. B. Langdon. Plasma Physics Via Computer Simulation. Institute of Physics Publishing, Bristol and Philadelphia, 2002.

[4] C. De Boor. A practical guide to splines, volume 27. Springer-Verlag, Berlin, Heidelberg, 2001.

[5] A. Bossavit. Mixed finite elements and the complex of Whitney forms. The Mathematics of Finite Elements and Applications VI, J. Whiteman (ed.), pages 137-144, 1988.

[6] Alain Bossavit. Computational electromagnetism and geometry : building a finite-dimensional "Maxwell's house" (part 5). J. Japan Soc. Applied Electromagnetism and Mechanics, 8:203-209, 2000.

[7] A. Buffa, J. Rivas, G. Sangalli, and R. Vzquez. Isogeometric analysis in electromagnetics: theory and testing. Preprint.

[8] A. Buffa, G. Sangalli, and R. Vazquez. Isogeometric analysis in electromagnetics: B-splines approximation. Comput. Methods Appl. Mech. Engrg, 199:1143-1152, 2009.

[9] E. Cohen, T. Martin, R.M. Kirby, T. Lyche, and R.F. Riesenfeld. Analysis-aware modeling: Understanding quality considerations in modeling for isogeometric analysis. Computer Methods in Applied Mechanics and Engineering, 199(5-8):334 - 356, 2010. Computational Geometry and Analysis.

[10] L Beirao da Veiga, A. Buffa, G. Sangalli, and J. Rivas. Some estimates for h/p/k refinement in isogeometric analysis. Technical Report IMATI-CNR n 20PV09/16/0.

[11] Ralf Hiptmair. Finite elements in computational electromagnetism. Acta Numerica, 11:237-339, 2002.

[12] Klaus Höllig. Finite Element Methods with B-Splines, volume 26. SIAM, 2003. Frontiers in Applied Mathematics.

[13] Klaus Höllig, Ulrich Reif, and Joachim Wipper. Weighted extended b-spline approximation of dirichlet problems. SIAM J. Numer. Anal., 39:442-462, February 2001.

[14] T.J.R. Hughes, J.A. Cottrell, and Y. Bazilevs. Isogeometric analysis: Cad, finite elements, nurbs, exact geometry and mesh refinement. Computer Methods in Applied Mechanics and Engineering, 194(39-41):4135 - 4195, 2005.

[15] T.J.R. Hughes, A. Reali, and G. Sangalli. Efficient quadrature for nurbs-based isogeometric analysis. Computer Methods in Applied Mechanics and Engineering, 199(5-8):301 - 313, 2010. Computational Geometry and Analysis.

[16] Y. Bazilevs J.A Cottrell, T. Hughes. Isogeometric Analysis, toward Integration of CAD and FEA. first ed., John Wiley \& Sons, Ltd, 2009

[17] T. Martin, E. Cohen, and R.M. Kirby. Volumetric parameterization and trivariate b-spline fitting using harmonic functions. Computer Aided Geometric Design, 26(6):648 - 664, 2009. Solid and Physical Modeling 2008, ACM Symposium on Solid and Physical Modeling and Applications.

[18] Ahmed Ratnani and Eric Sonnendrücker. Arbitrary high-order spline finite element solver for the time domain maxwell equations. J. Sci. Comput. to appear.

[19] E. Sonnendrücker. Modèles cinétiques pour la fusion. notes du cours de M2 (2008).

[20] G. Xu, B. Mourrain, R. Duvigneau, and A. Galligo. Optimal analysis-aware parameterization of computational domain in isogeometric analysis. submitted. 


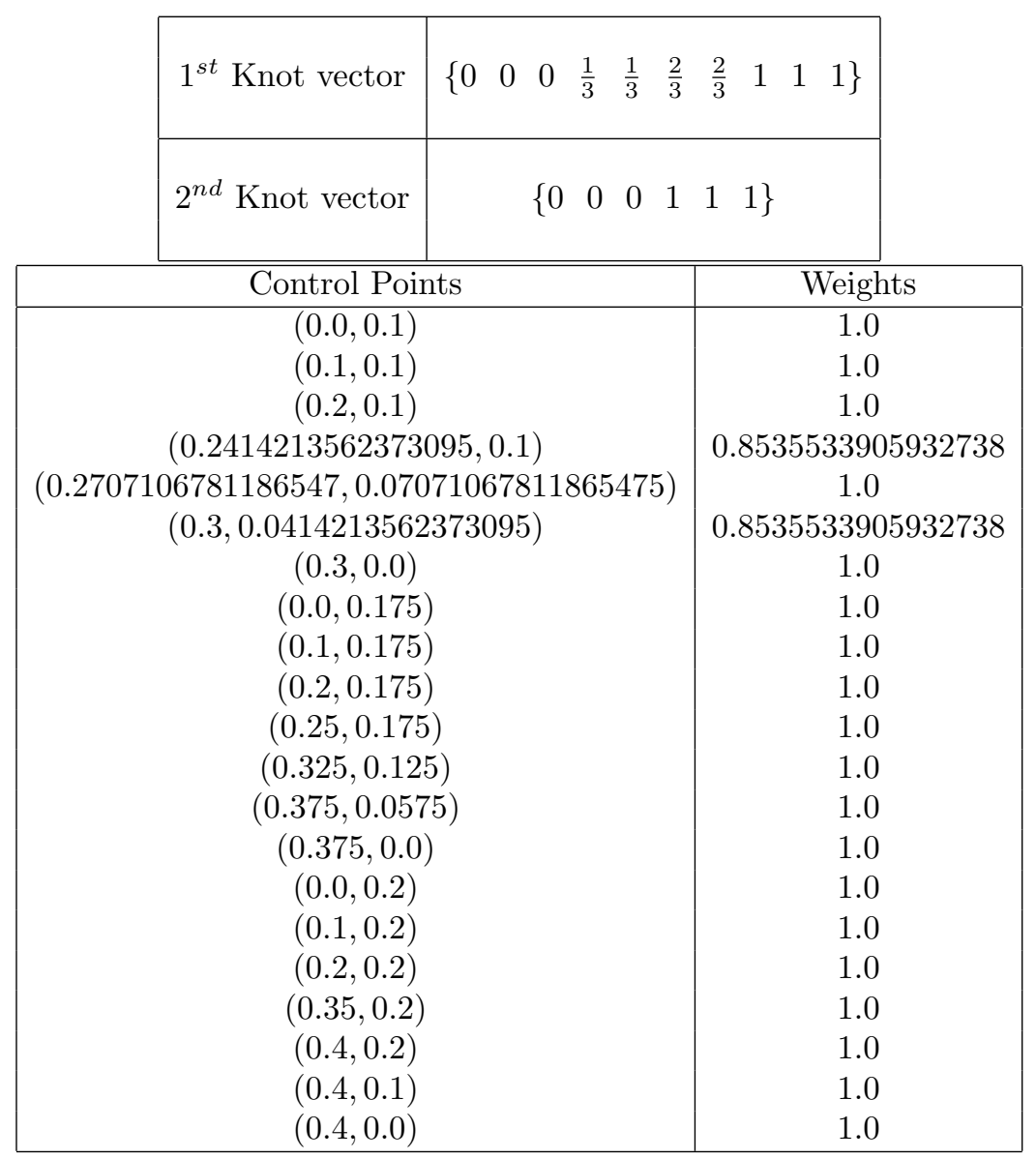

Figure 8. Diode description using NURBS. 